\title{
The Research on Earthquake Radon Anomalies
}

\author{
Liangquan Ge, Jiankun Zhao, Yaoyao Luo \\ College of Applied Nuclear Technology and Automation Engineering, Chengdu University of Technology, \\ Chengdu, China \\ Email: gla@cdut.edu.cn
}

Received September 2014

\begin{abstract}
Radon is considered to be one of the most promising gases to predicting the earthquake. The water radon concentration is regular to monitoring indicator. The research on the formation of the earthquake radon anomalies (ERA) will produce great academic value and economic benefits. The ERA in slope-type can be seemed as a response of underground water radon anomalies in the progress of tensile stress accumulating.
\end{abstract}

\section{Keywords}

The Earthquake Radon, The Water Radon Anomalies, Earthquake Prediction

\section{Introduction}

To make the relationship between earthquake and radon anomaly clear, the Chinese seismologists had done a lot of related research in radon and thoron exhalations during fracture in 1970s. Luo Guangwei proposed that radon concentration increased significantly under the uniaxial pressure (Luo, 1980). Based on the data of the AMRITASR Earthquake Monitoring Station of India, Han Xuehui of TYUT present some neodoxy in 2000 as followed: after a new round of tectonic stress action, geologic body will be to experience "compaction stage" firstly, and at this stage the tectonic stress impact on geologic body increase gradually, the primary fracture will decrease with the increase of stress until to be closed, which shut down the original radon exhalation channel. With all of these reasons, the radon concentration will present downward obviously at this stage (Han, 2000). After the Wenchuan Ms 8.0 earthquake, Gu Yi of Chengdu University of Technology (CDUT) evaluated the earthquake fault activity by measuring the soil radon concentration on the activity fault section, and the results show that the method is effective and reliable ( $\mathrm{Gu}, 2009)$.

\section{The Forming Mechanism of Earthquake Radon Anomalies}

In the process of earthquake preparation, the underground rock in seismogenic zone also undergoes the experiences above the stages. When the rock cannot expand any more, the tiny fractures will cluster to the main rupture leading to the earthquake swarm which is regarded as the precursor for megaseism (QIN, 2011). In the initial accumulation stage, the connectivity between inner pores become worse and pore size gets smaller. It not 
only reduces the effective space of the storage for radon, but also blocks the migration of free radon in pores and fractures. Therefore, in the process of stress accumulation, the free radon concentration in the pores or fractures will be reduced which is confirmed in the experiments. When the rock under continuous stress or greater stress, the porosity increases significantly, so does the pore connectivity. This not only increases the free radon concentration in the pore, but also drives the migration of radon easily which is conducive to the detection.

When the rock undergoes a tensile stress for longtime, the crack of rock will expand. Obviously, rock porosity and pore connectivity will continue to increase, which is conducive to increase the free radon concentration in rock pore and to easily migrate.

As a result, no matter compression, stretching, or deformation, the free radon concentration in rock pores will change and an Earthquake Radon Anomaly (ERA) will form in seismogenic zone. Based on the ERA, we not only may predict the changes of rock stress at the monitoring plots, but also indicate the types of stress loaded on the rocks, such as compression is tension stress.

\section{The Discussion of ERA Morphology}

In the process of seismogenic, the ERA morphology is related to many factors including the monitoring area, measuring objects and the sensitivity of instruments. At present, the object of long-term monitoring radon is groundwater. Since phreatic fractures and pores are fully filled of water, the radon emits from rocks and dissolves in underground water immediately. Groundwater becomes the carrier of radon migration. So, the ERA is closely relative to underground water movement. According to the behaviors of radon in the process of seismogenic, the morphology of ERA can be expressed as "slope style", "hook style" and "pulse style", shown in Figure 1.

The slope type of ERA (Figure 1(a)) is forming in seismogenic stretch area. In this area, tensile stress forces on rocks and the tiny fractures in rocks may increase and expand gradually, which is in favor of the storage and space for radon migration. The radon concentration in groundwater will gradually increase. In the process of rack sustained compression, the "slope style" anomaly may develop in multistep.

The hook style of ERA (Figure 1(b)) may be observed in the seismogenic compressive area. The ERA consists of descent stage and ascent stage. The descent stage represents the initial compaction and elastic deformation. In this stage, the fractures are compressed and the path of radon migration may be closed, which leads to reduce radon concentration in groundwater gradually. The ascent stage of ERA shows that cracks grow crazily and rocks break. In this stage, porosity and pore connectivity increase fast, so does the concentration. The rate of ascent is significantly faster than that of descent.

The pulse style (Figure 1(c)) describes the radon concentration in underground water increases and decrease rapidly. Its intensity is strong, also can be superimposed on the "slope style" or "hook style". This kind of

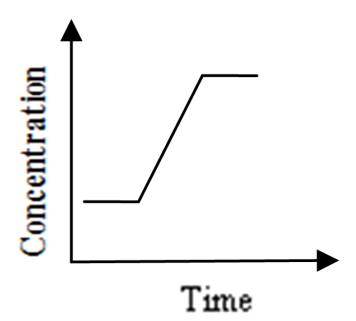

(a)

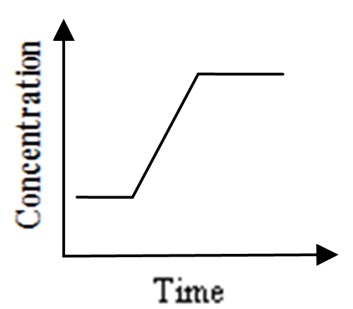

(b)

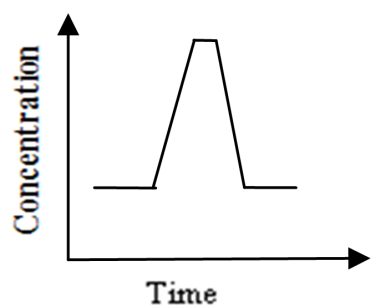

(c)

Figure 1. The patterns of ERA (a) slope-type; (b) hook-type; (c) pulse-type. 
anomaly may be caused by a alternant deformation to expand the porosity or the radon releases from the rock fitfully.

\section{The Discussion about ERA in Time-Space Domain}

As the half-life of ${ }^{222} \mathrm{Rn}$ is only 3.825 days, the diffusion length of radon in water is just a few centimeters. For this reason, the range of a radon anomaly in underground water is strongly depended on the movement of under groundwater. Since the radioactive decay equilibrium between ${ }^{222} \mathrm{Rn}$ and ${ }^{226} \mathrm{Ra}$ in a closed water-rock system takes 38 days that is about 10 times half-time of ${ }^{222} \mathrm{Rn}$, the range of ERA should cover the distance of underground water movement in 38 days.

The time span of ERA is the whole time of rock stress accumulating. To the short-time "slope type" pattern caused by single compression change, it takes 38 days to balance the radium-radon decay. For the long time tensile stress accumulating, the "slope style" anomaly develops in multistep. It will impact the whole process of sustained compression. The descent stage of "hook style" anomaly may decline in multistep; the ascent stage presents rock broken superimposed many times. The "pulse style" anomaly caused by radon released fitfully takes very short time to be formed. According to the monitoring data to free radon released from soil, the pulse style is measured in units of hours or days. While it caused by a rock alternant deformation, the anomaly will sustain for longer time. In addition, the more "pulse style" anomalies appear the more tremendously stress change will happen near the monitoring station.

\section{Conclusion}

In the process of seismogenic, no matter rocks endure compression, stretching, or deformation, the free radon concentration in rock pores will change and an Earthquake Radon Anomaly (ERA) will form in seismogenic zone. The morphology of ERA can be described as "slope-type”, "hook-type” and "pulse-type”. The slope type represents the underground radon anomaly in the seismogenic stretch area. The ERA can cover where the underground water with radon moves in 38 days and go through the whole time of rock sustained compression. The ERA can reflect the states the rock in such as stretch, compression and broken. So the monitoring station should be located not only tensile area but also compressive zone. By analyzing the regional ERA comprehensively, we provide important reference information for seismology and earthquake prediction.

\section{Acknowledgements}

The authors gratefully acknowledge the financial support of: (1) National Natural Science Foundation of China through Grant No. 41074093 entitled "Research on mechanism of earthquake radon anomaly". (2) The National High-tech Research and Development Program of China through Grant 2012AA061803 entitled "Development of high precision nuclear radiation spectrometry”.

\section{References}

Luo, G. W., \& Shi, X. Z. (1980). Experimental Results of Radon and Thorium Emanations from Rock Specimen under Pressure. Acta Seismologica Sinica, 2, 198-204.

Han, X. H. (2000). The Ideal Curve Model on Earthquake Prediction by Radon Monitoring. Journal of Taiyuan University of Technology, 31, 101-103.

Gu, Y (2009).Analysis and Evaluation of Faults Activities in Chengdu Region with Radon Concentration Measurements after Wenchuan Earthquake. Journal of Engineering Geology, 17, 296-300.

Qin, S. Q. (2011). Seismogenic Law and Mode of Strong Earthquakes. Journal of Earth Sciences and Environment, 33, 311316. 